\title{
Research Letter \\ Surface Passivation and Photoluminescence of Mn-Doped ZnS Nanocrystals
}

\author{
Ping Yang and Michael Bredol \\ Department of Chemical Engineering, Fachhochschule Münster University of Applied Sciences, Stegerwaldstrasse 39, \\ Steinfurt 48565, Germany
}

Correspondence should be addressed to Michael Bredol, bredol@fh-muenster.de

Received 12 February 2008; Accepted 21 March 2008

Recommended by William A. Jesser

Enhanced photoluminescence (PL) is reported from Mn-doped ZnS nanocrystals (NCs) capped with ZnS (ZnS:Mn/ZnS core-shell NCs) and thioglycolic acid (TGA) (ZnS:Mn/ZnS core-shell NCs dispersed in an alkaline TGA solution). The NCs were prepared using a reverse micelle route. Comparing with initial ZnS:Mn core NCs, the ZnS:Mn/ZnS core-shell NCs exhibit much stronger orange PL $(\sim 580 \mathrm{~nm})$. This is presumably the result of effective passivation of quenching ZnS:Mn NCs surface states by a pure $\mathrm{ZnS}$ shell. As for TGA-capped ZnS:Mn/ZnS core-shell NCs, the parallel decrease of a defect-related emission of ZnS is associated with the formation of a shell surface layer of TGA-Zn complexes. In summary, the combination of ZnS shells with TGA ligands was demonstrated to yield ZnS:Mn NCs with narrow size distribution and intense PL.

Copyright (c) 2008 P. Yang and M. Bredol. This is an open access article distributed under the Creative Commons Attribution License, which permits unrestricted use, distribution, and reproduction in any medium, provided the original work is properly cited.

\section{INTRODUCTION}

Doped semiconductor nanocrystals (NCs) have been studied extensively in the past few decades since Bhargava [1] reported that $\mathrm{ZnS}: \mathrm{Mn}^{2+} \mathrm{NCs}$ exhibit high-luminescence quantum efficiency in 1994. Mn-doped ZnS is widely recognized as an efficient phosphor, especially, when it is used as a phosphor material for electroluminescent displays [2]. Therefore, Mn-doped NCs have attracted much attention in recent years [3-9]. Nanosized and thus optically transparent emitters for electroluminescent displays made by simple thick film technologies (e.g., printing) would offer exciting new capabilities for industrial application of these nanomaterials in lamps or displays, if present issues with efficiency and life time could be overcome.

The photoluminescence (PL) intensity of Mn-doped ZnS NCs is depending on the $\mathrm{Mn}^{2+}$ concentration $[10,11]$. In addition, especially in NCs the elimination of structural defects plays an important role to enhance the luminescence of $\mathrm{Mn}^{2+}$, they are known as luminescence quenching sites and play a role in photodegradation. The surface states are likely to trap electrons and/or holes, inducing nonradiative recombination. Thus more effective surface passivation me- thods have been an important objective and should lead to more efficient PL and photostability. Bol and Meijerink investigated, for example, the influence of $\mathrm{Mn}^{2+}$ concentration and a passivating polymer on the luminescence quantum efficiency of ZnS:Mn NCs [5]. Yang and Holloway reported on highly luminescent and photostable yellowemitting CdS:Mn/ZnS core-shell NCs [12]. They indicated that the intense PL of the NCs is based on the effective passivation of CdS:Mn core surface states by a $\mathrm{ZnS}$ shell. Kim et al. observed surface modification effects on the PL properties of CdS and CdS:Mn NCs [13]. Furthermore, enhanced PL is also observed when Mn-doped ZnS NCs are capped with an effective passivation layer, such as $\mathrm{ZnO}$ [14] or $\mathrm{SiO}_{2}$ [15]. These inorganic capping agents saturate the surface dangling bonds and substantially reduce the nonradiative centers of the NCs. When dispersed in liquids, solvent molecules can act as passivators as well. Cordero et al. [16] suggested that the water molecules adsorbed on CdSe NCs surface act to fill surface trap sites, leading to enhanced luminescence.

The reverse micelle route is an established technique to prepare NCs with a narrow size distribution. The NCs are generated inside the water pools of the micellar system and 
thus size-selective NCs with narrow size distribution can be obtained [17]. Additionally, it is quite easy to modify the surface of the NCs during reverse micellar preparation with a shell material. Core/shell NCs usually reveal high $\mathrm{PL}$ if the shell layer has a wider band gap than the core NCs. For example, Santra et al. prepared highly waterdispersible, multifunctional, CdS:Mn/ZnS core-shell NCs using a water-in-oil microemulsion method [18]. In this article, we describe $\mathrm{ZnS}: \mathrm{Mn} / \mathrm{ZnS}$ core-shell NCs with narrow size distribution via a reverse micelle route.

Apart from surface passivation, another challenge is the dispersibility of NCs. Many organic capping agents were already tried to reduce agglomeration of the NCs and increase PL during preparation and dispersion. In this regard, thioglycolic acid (TGA) is an effective capping agent to prepare CdTe [19, 20] and CdSe [21] NCs because the thiole group in TGA can link effectively to the $\mathrm{Cd}^{2+}$ ion on the surface of the NCs. This leads to efficient surface passivation. Thiole-capped CdTe colloidal solution has revealed high-PL quantum efficiency (65\%) using TGA as a capping agent [20]. Furthermore, the PL quantum efficiency of these TGAstabilized NCs reached up to $85 \%$ after illumination, as reported by Gao's group [19]. Therefore, we investigated the effect of the concentration and $\mathrm{pH}$ of TGA solutions on the dispersion because TGA is stable in alkaline solution [22].

\section{EXPERIMENTAL}

All chemicals used were of analytical grade or of the highest purity available. Milli-Q water was used as a solvent. Zinc acetate dihydrate $\left(\mathrm{Zn}\left(\mathrm{CH}_{3} \mathrm{COO}\right)_{2} \cdot 2 \mathrm{H}_{2} \mathrm{O}, 98+\%\right)$, manganese acetate tetrahydrate $\left(\mathrm{Mn}\left(\mathrm{CH}_{3} \mathrm{COO}\right)_{2} \cdot 4 \mathrm{H}_{2} \mathrm{O}, 99+\%\right)$, sodium sulfide nonahydrate $\left(\mathrm{Na}_{2} \mathrm{~S} \cdot 9 \mathrm{H}_{2} \mathrm{O}, 98+\%\right)$, polyoxyethylene(5)nonylether (Igepal CO-520, 99.9+\%), cyclohexane, and TGA were purchased from Sigma Aldrich, St. Louis, USA.

\subsection{Preparation of ZnS:Mn/ZnS core-shell NCs}

$\mathrm{Zn}^{2+}-,\left(\mathrm{Zn}^{2+}+\mathrm{Mn}^{2+}\right)$-, and $\mathrm{S}^{2-}$-containing stock solutions $(0.5 \mathrm{M})$ were prepared by dissolving $\mathrm{Zn}\left(\mathrm{CH}_{3} \mathrm{COO}\right)_{2} \cdot 2 \mathrm{H}_{2} \mathrm{O}$, $\mathrm{Mn}\left(\mathrm{CH}_{3} \mathrm{COO}\right)_{2} \cdot 4 \mathrm{H}_{2} \mathrm{O}$, and $\mathrm{Na}_{2} \mathrm{~S} \cdot 9 \mathrm{H}_{2} \mathrm{O}$ in water. The molar ratio of $\mathrm{Mn}$ to $\mathrm{Zn}$ was set to the desired value. Each solution was mixed with an Igepal CO-520/cyclohexane stock solution, with the concentration of Igepal CO-520 in the microemulsion set to $0.24 \mathrm{M}$. The water-to-surfactant molar ratio $\mathrm{W}$ for the formation of the $\mathrm{ZnS}: \mathrm{Mn} / \mathrm{ZnS}$ core-shell NCs was 3.7. ZnS:Mn core NCs were obtained by injecting first $5 \mathrm{~mL} \mathrm{~S}^{2-}$-containing microemulsion to the $\left(\mathrm{Zn}^{2+}+\mathrm{Mn}^{2+}\right)$ containing microemulsion within 2 minutes, followed by further vigorous stirring for 15 minutes. Then $48 \mathrm{~mL}$ $\mathrm{S}^{2-}$-containing microemulsion was injected at a rate of $0.5 \mathrm{~mL} / \mathrm{min}$ into above microemulsion followed by stirring for 60 minutes.

A $\mathrm{ZnS}$ shell layer was grown on the $\mathrm{ZnS}: \mathrm{Mn}$ core $\mathrm{NCs}$ using $0.5 \mathrm{M}$ solutions of $\mathrm{Zn}\left(\mathrm{CH}_{3} \mathrm{COO}\right)_{2} \cdot 2 \mathrm{H}_{2} \mathrm{O}$ and $\mathrm{Na}_{2} \mathrm{~S} \cdot 9 \mathrm{H}_{2} \mathrm{O}$ prepared as described above. $\mathrm{Zn}^{2+}$ or $\mathrm{S}^{2-}$ ions aqueous solutions were mixed with the Igepal CO$520 /$ cyclohexane stock solution with the same W. A $\mathrm{S}^{2-}$ - containing microemulsion was first injected at a rate of $0.5 \mathrm{~mL} / \mathrm{min}$ into the $\mathrm{ZnS}: \mathrm{Mn} \mathrm{NCs}$ microemulsion and then stirred rapidly for 30 minutes. A $\mathrm{Zn}^{2+}$-containing microemulsion was then injected at a very slow rate of $0.2 \mathrm{~mL} / \mathrm{min}$ and then stirred rapidly for 60 minutes. The unwanted nucleation and growth of a separated $\mathrm{ZnS}$ phase was suppressed by maintaining a slow addition rate of the $\mathrm{Zn}^{2+}$ microemulsion [23]. The molar ratio of $\mathrm{ZnS}$ to $\mathrm{ZnS}: \mathrm{Mn}$ in the microemulsion was $0.15-0.40$ to investigate the effect of the thickness of $\mathrm{ZnS}$ shell. Because anion dangling bonds on the surface of NCs result in decreased luminescence $[12,19]$, the amount of $\mathrm{Zn}^{2+}$ is in excess compared with that of $S^{2-}$ in the microemulsion. To provide optimal reproducibility, in each of the series, the samples were prepared twice, always at the same time and from the same stock solutions, keeping all reaction conditions the same.

\subsection{Dispersion and surface passivation of the NCs}

Anhydrous acetone was first added to the microemulsion to precipitate the NCs. The NCs were then centrifuged, washed thoroughly using anhydrous acetone, centrifuged again to eliminate surfactant, then washed thoroughly by a $0.05 \mathrm{M}$ $\mathrm{Zn}^{2+}$ solution to eliminate acetone and form a nearly $\mathrm{S}^{2-}$ free surface on the NCs. After this treatment, they were dispersed in an aqueous solution of TGA with different TGA concentrations $(0.01 \sim 0.05 \mathrm{M})$ and $\mathrm{pH}(7 \sim 10)$ adjusted using a $0.5 \mathrm{M} \mathrm{NaOH}$ solution.

\subsection{Characterization}

Emission and excitation spectra were recorded with a Shimadzu RF-5301PC fluorescence spectrometer. Powder X-ray diffraction (XRD) was taken with a Philips diffractometer. The size distribution of the samples was measured by dynamic light scattering (DLS, Malvern high-performance particle sizer).

\section{RESULTS}

The XRD pattern of the ZnS:Mn/ZnS core-shell NCs revealed zinc blende structure. Because individual $\mathrm{Mn}$ impurities are most easily incorporated in NCs with zincblende structure by adsorbing on their (001) facet [4], ZnS NCs with zinc-blende structure are most desirable to enhance $\mathrm{Mn}^{2+}$ emission. The mean size of the core/shell-NCs dispersed in TGA is $\sim 11 \mathrm{~nm}$, according to DLS.

Figure 1(a) shows photographs of the $\mathrm{ZnS}: \mathrm{Mn}$ core and $\mathrm{ZnS}: \mathrm{Mn} / \mathrm{ZnS}$ core-shell NCs in microemulsion upon $366 \mathrm{~nm}$ UV and room light illumination. Figure 1(b) shows typical emission and excitation spectra of the $\mathrm{ZnS}: \mathrm{Mn}$ core and $\mathrm{ZnS}: \mathrm{Mn} / \mathrm{ZnS}$ core-shell NCs in microemulsion. While the ZnS:Mn/ZnS core-shell NCs exhibit significant $\mathrm{Mn}^{2+}$ emission, the $\mathrm{ZnS}: \mathrm{Mn}$ NCs show negligible $\mathrm{Mn}^{2+}$ emission. To investigate the influence of $\mathrm{Mn}^{2+}$ on the PL of the ZnS:Mn NCs, we observed the PL spectra of the $\mathrm{ZnS}: \mathrm{Mn} \mathrm{NCs}$ with different $\mathrm{Mn}^{2+}$ ratio (1-8\%). Highest PL intensity was found with a molar fraction of $4-5 \%$; no 


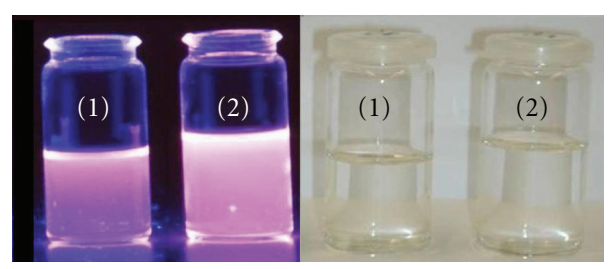

(a)

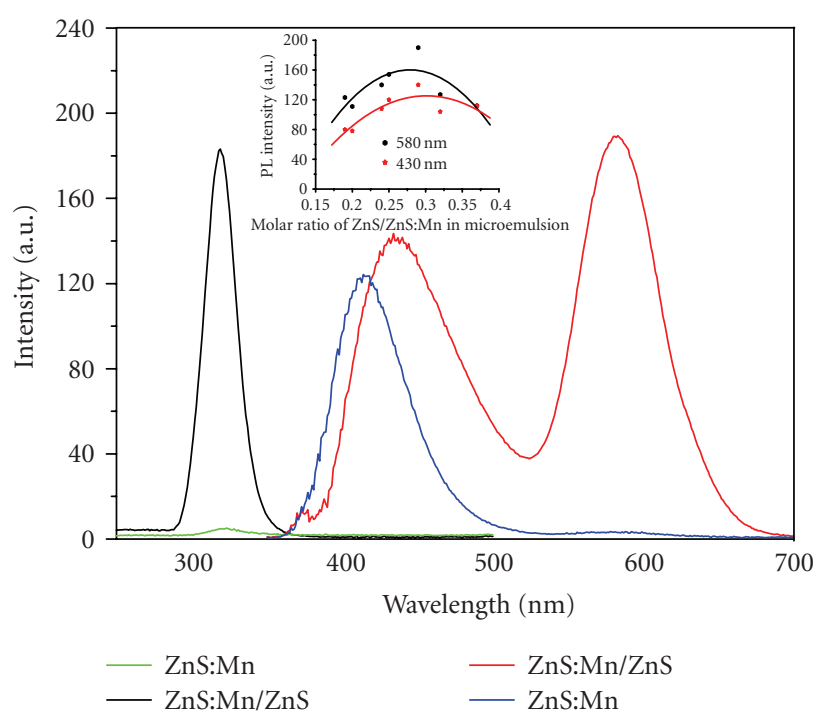

(b)

Figure 1: (a) Photographs of ZnS:Mn core NCs (1) and ZnS: $\mathrm{Mn} / \mathrm{ZnS}$ core-shell NCs (2), after preparation in microemulsion upon $366 \mathrm{~nm}$ UV (left) and day light (right) illumination. (b) Excitation and PL spectra of ZnS:Mn and ZnS:Mn/ZnS NCs in microemulsion. Inset: PL intensity of $\mathrm{ZnS}: \mathrm{Mn} / \mathrm{ZnS}$ core-shell NCs at $580 \mathrm{~nm}$ and $430 \mathrm{~nm}$ versus molar ratio of $\mathrm{ZnS} / \mathrm{ZnS}: \mathrm{Mn}$.

change on position of excitation and emission peaks was observed. The enhanced emission of the $\mathrm{ZnS}: \mathrm{Mn} / \mathrm{ZnS}$ coreshell NCs strongly depends on the thickness of the $\mathrm{ZnS}$ shell. Efficient passivation will take place when the molar ratio of $\mathrm{ZnS} / \mathrm{ZnS}: \mathrm{Mn}$ in the precursor microemulsion is $0.25-0.30$ (inset in Figure 1(b)).

Figure 2 shows the excitation and PL spectra of the ZnS:Mn/ZnS core-shell NCs dispersed in an alkaline TGA solution with different TGA concentrations and $\mathrm{pH}$. The sharp excitation spectrum reveals that the NCs exhibit a widened, but well-defined band gap which means a narrow size distribution. Comparing with their initial spectra in microemulsion, the defect-related emission was decreased and $\mathrm{Mn}^{2+}$ emission was increased. The PL intensity of the NCs dispersed in an alkaline TGA solution depends strongly on the $\mathrm{pH}$ and concentration of the TGA solutions (inset in Figure 2). When the concentration of TGA ( $\mathrm{pH} 9.1)$ is higher than $0.035 \mathrm{M}$, the PL intensity of $\mathrm{Mn}^{2+}$ decreased and defect-related emission was increased slightly. Presumably, this is caused by a deterioration of the surface due to a partial dissolution by the TGA under these conditions.

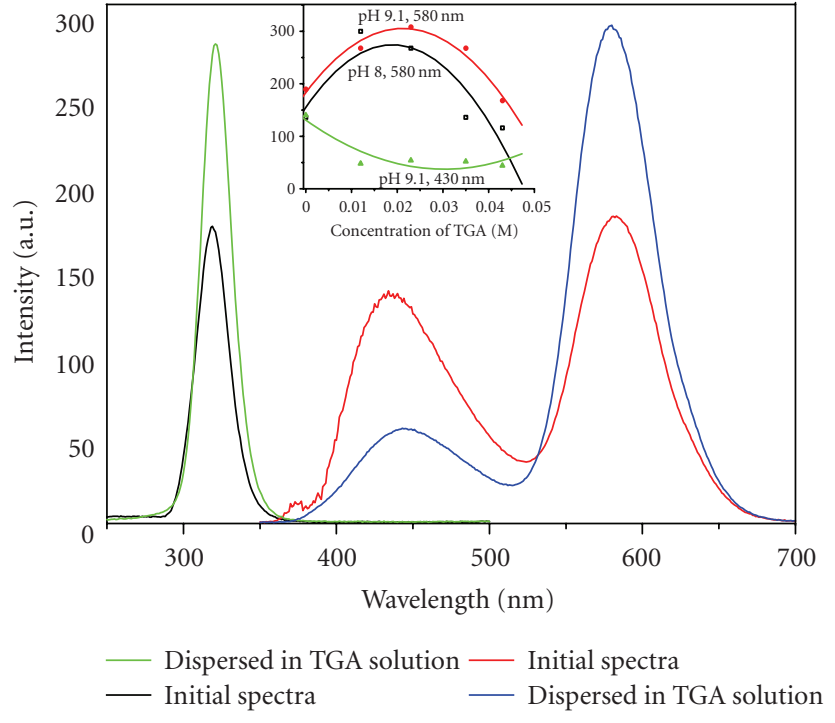

FIgure 2: Excitation and PL spectra of ZnS:Mn/ZnS core-shell NCs dispersed in a TGA solution $(0.023 \mathrm{M}, \mathrm{pH}$ 9.0). Inset: PL intensity of ZnS:Mn/ZnS core-shell NCs at $580 \mathrm{~nm}$ and $430 \mathrm{~nm}$ against concentration of TGA with different $\mathrm{pH}$. Initial excitation and emission spectra of $\mathrm{ZnS}: \mathrm{Mn} / \mathrm{ZnS}$ core-shell NCs in microemulsion are shown for comparison.

Figure 3(a) shows photographs of $\mathrm{ZnS}: \mathrm{Mn} / \mathrm{ZnS}$ coreshell NCs dispersed in water and in TGA solution upon $366 \mathrm{~nm}$ UV and room light illumination. Figure 3(b) shows the $\mathrm{pH}$ dependence on dispersing the NCs into TGA solutions. The NCs in this case showed no agglomeration and a transparent solution was obtained. At the experimental TGA concentration $(0.035 \mathrm{M}$, the NCs cannot be monodispersed completely when $\mathrm{pH}$ is less than 7.8, and the $\mathrm{Mn}^{2+}$ emission of the NCs in a TGA solution decreases when $\mathrm{pH}$ is higher than 8.5. The optimal compromise for monodispersed NCs with high PL was demonstrated to be a concentration of TGA of $0.015-0.03 \mathrm{M}$ and a $\mathrm{pH}$ in the range 8-9.

\section{DISCUSSION}

The ZnS:Mn NCs exhibit a violet/blue emission and an orange emission upon $315 \mathrm{~nm}$ excitation. The violet/blue emission is associated with a defect-related emission of the $\mathrm{ZnS}$ host, and orange emission is attributed to the ${ }^{4} T_{1} \rightarrow{ }^{6} A_{1}$ transition of the $\mathrm{Mn}^{2+}$ ion. Compared with $\mathrm{Mn}$-doped $\mathrm{ZnS}$ NCs prepared by standard methods [5] (coprecipitation in aqueous solution at $80 \sim 100^{\circ} \mathrm{C}$ ), the defect-related emission is stronger from the as-prepared $\mathrm{ZnS}: \mathrm{Mn}$ NCs because the preparation was carried out at room temperature. Therefore, $\mathrm{Mn}^{2+} \mathrm{PL}$ of unpassivated ZnS:Mn NCs is weak. The intensity of defect-related emission depends strongly on the ratio of $\mathrm{Mn}^{2+}$ in solution. As soon as $\mathrm{Mn}^{2+}$ is incorporated in $\mathrm{ZnS} \mathrm{NCs}$, the defect-related emission decreases and $\mathrm{Mn}^{2+}$ emission comes up because the energy transfer between $\mathrm{ZnS}$ host and $\mathrm{Mn}^{2+}$ impurity is very efficient.

An inorganic shell passivation on the NCs not only leads to a significant reduction of surface-related states, but also 


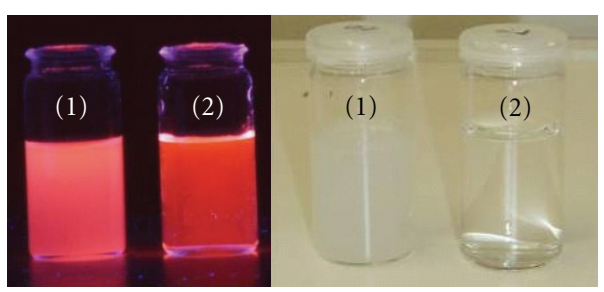

(a)

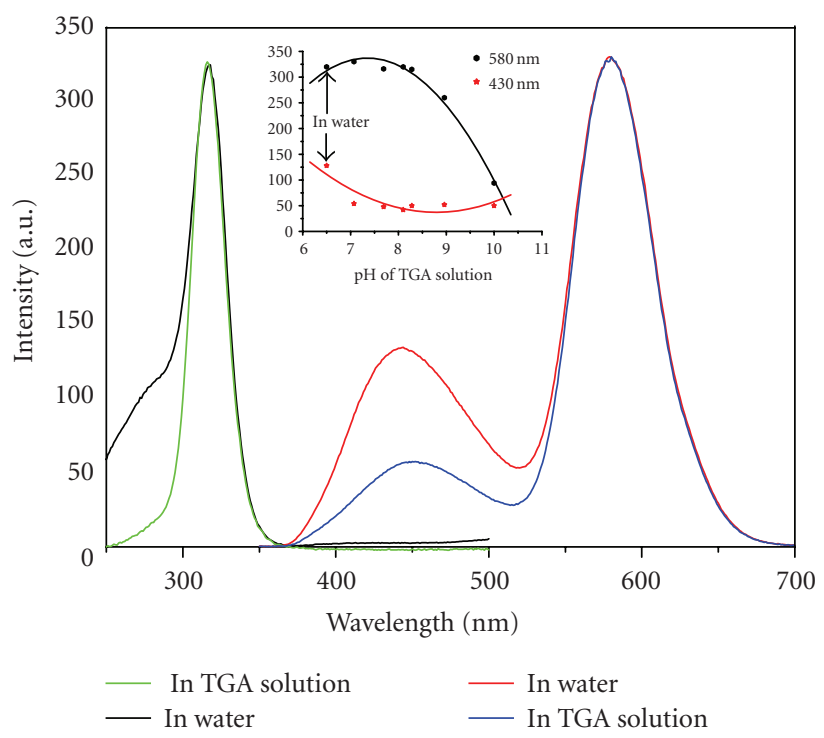

(b)

Figure 3: (a) Photographs of ZnS:Mn/ZnS core-shell NCs dispersed in water (1) and a TGA solution (2) upon $366 \mathrm{~nm} \mathrm{UV}$ (left) and day light (right) illumination. (b) Excitation and PL spectra of $\mathrm{ZnS}: \mathrm{Mn} / \mathrm{ZnS}$ core-shell NCs dispersed in a TGA solution (0.035 M, pH 8.5). Inset: PL intensity of $\mathrm{ZnS}: \mathrm{Mn} / \mathrm{ZnS}$ core-shell $\mathrm{NCs}$ at $580 \mathrm{~nm}$ and $430 \mathrm{~nm}$ against $\mathrm{pH}$ of TGA solutions $(0.035 \mathrm{M})$. Excitation and emission spectra of $\mathrm{ZnS}: \mathrm{Mn} / \mathrm{ZnS}$ core-shell NCs dispersed in an aqueous solution with same NCs concentration are shown for comparison.

leads to the confinement of charge carriers in the core region due to band offset potentials (the very thin shell should have a band gap even larger than the slightly larger core), resulting in more efficient and photostable luminescence. After passivating by a $\mathrm{ZnS}$ shell, the emission from $\mathrm{Mn}^{2+}$ $(580 \mathrm{~nm})$ was dramatically enhanced. The formation of the $\mathrm{ZnS}$ shell was carried out with a slow injecting rate in order to achieve a crystallinity as high as possible in the shell. However, the defect-related emission is still observed after passivation. This result differs from the report by Yang and Holloway on ZnS-passivated CdS:Mn NCs [12], where no defect-related emission was observed after passivation. The difference between the behavior of CdS:Mn and ZnS:Mn NCs originates most probably from the different band gap differences of core and shell and thus a stronger tendency to confinement in CdS:Mn NCs.

In general, TGA serves well as stabilizer to prepare CdTe [20] and CdSe [21] NCs. CdTe NCs prepared this way possess negative surface charge because of the surface carboxylic groups in TGA and exhibit efficient luminescence for months and even years being stored under air in the dark at room temperature. The ZnS:Mn/ZnS core-shell NCs have similar surface states as compared to CdTe NCs. The ZnS:Mn/ZnS core-shell NCs possess a surface rich in zinc and nearly free of $\mathrm{S}^{2-}$ ions because the NCs were washed with a $\mathrm{Zn}^{2+}$ solution before dispersion. The $\mathrm{Zn}^{2+}$ ions on the surface of the NCs can be coordinated with the mercapto groups in TGA during dispersion. The carboxyl group of TGA then is presented on the outer surface of the NCs in an alkaline solution. Depending on $\mathrm{pH}$, carboxyl groups tend to dissociate to form $\left(-\mathrm{COO}^{-}\right)$[22]. Therefore, the surface of TGA-capped NCs has negative charge, preventing the NCs from agglomeration. For TGA-capped emitting NCs, a shell of thiolate complexes is probably formed at the surface of the NCs, passivating dangling bonds, but without possibility of energy transfer to it. This is a situation in analogy to, for example, a $\mathrm{ZnS}$ shell on CdSe [21]. Therefore, a strong enhancement of PL was observed from TGA-capped emitting NCs.

\section{CONCLUSIONS}

The preparation of $\mathrm{ZnS}: \mathrm{Mn}$ and $\mathrm{ZnS}: \mathrm{Mn} / \mathrm{ZnS}$ core-shell NCs via a reverse micelle route has been described. A $\mathrm{ZnS}$ shell leads to efficient passivation of the ZnS:Mn core when the molar ratio of $\mathrm{ZnS} / \mathrm{ZnS}: \mathrm{Mn}$ in the precursor microemulsion is $0.25-0.30$. The $\mathrm{ZnS}: \mathrm{Mn} / \mathrm{ZnS}$ core-shell NCs reveal zincblende structure and narrow size distribution (mean size $11 \mathrm{~nm}$ ). The PL and dispersion of the $\mathrm{ZnS}: \mathrm{Mn} / \mathrm{ZnS}$ core-shell NCs in an alkaline TGA solution depends strongly on the concentration of TGA and $\mathrm{pH}$. A monodispersed colloidal solution of the NCs with high PL is obtained when the concentration of TGA is $0.015-0.03 \mathrm{M}$ and $\mathrm{pH}$ is $8-9$.

\section{ACKNOWLEDGMENTS}

Thanks are due to Thomas Jüstel, Ulrich Kynast, and their groups (all at FH Münster) for the possibility to perform optical measurements. The work was funded by Bundesland NRW in the framework of the Kompetenzplattform Nanoskalige Materialien.

\section{REFERENCES}

[1] R. N. Bhargava, D. Gallagher, X. Hong, and A. Nurmikko, "Optical properties of manganese-doped nanocrystals of ZnS," Physical Review Letters, vol. 72, no. 3, pp. 416-419, 1994.

[2] J. K. Furdyna, "Diluted magnetic semiconductors," Journal of Applied Physics, vol. 64, no. 4, pp. R29-R64, 1988.

[3] H. Yang, P. H. Holloway, and B. B. Ratna, "Photoluminescent and electroluminescent properties of Mn-doped $\mathrm{ZnS}$ nanocrystals," Journal of Applied Physics, vol. 93, no. 1, pp. 586-592, 2003.

[4] S. C. Erwin, L. Zu, M. I. Haftel, A. L. Efros, T. A. Kennedy, and D. J. Norris, "Doping semiconductor nanocrystals," Nature, vol. 436, no. 7047, pp. 91-94, 2005. 
[5] A. A. Bol and A. Meijerink, "Luminescence quantum efficiency of nanocrystalline $\mathrm{ZnS}: \mathrm{Mn}^{2+}$. 1 . Surface passivation and $\mathrm{Mn}^{2+}$ concentration," Journal of Physical Chemistry B, vol. 105, no. 42, pp. 10197-10202, 2001.

[6] F. H. Su, Z. L. Fang, B. S. Ma, K. Ding, G. H. Li, and W. Chen, "Pressure dependence of $\mathrm{Mn}^{2+}$ luminescence in differently sized ZnS:Mn nanoparticles," Journal of Physical Chemistry B, vol. 107, no. 29, pp. 6991-6996, 2003.

[7] N. Karar, F. Singh, and B. R. Mehta, "Structure and photoluminescence studies on ZnS:Mn nanoparticles," Journal of Applied Physics, vol. 95, no. 2, pp. 656-660, 2004.

[8] P. A. Gonzalez Beermann, B. R. McGarvey, S. Muralidharan, and R. C. W. Sung, "EPR spectra of $\mathrm{Mn}^{2+}$-doped $\mathrm{ZnS}$ quantum dots," Chemistry of Materials, vol. 16, no. 5, pp. 915-918, 2004.

[9] S. Sapra, A. Prakash, A. Ghangrekar, N. Periasamy, and D. D. Sarma, "Emission properties of manganese-doped ZnS nanocrystals," Journal of Physical Chemistry B, vol. 109, no. 5, pp. 1663-1668, 2005.

[10] A. A. Khosravi, M. Kundu, B. A. Kuruvilla, et al., "Manganese doped zinc sulphide nanoparticles by aqueous method," Applied Physics Letters, vol. 67, no. 17, pp. 2506-2508, 1995.

[11] K. Sooklal, B. S. Cullum, S. M. Angel, and C. J. Murphy, "Photophysical properties of ZnS nanoclusters with spatially localized $\mathrm{Mn}^{2+}$," Journal of Physical Chemistry, vol. 100, no. 11, pp. 4551-4555, 1996.

[12] H. Yang and P. H. Holloway, "Efficient and photostable ZnS-passivated CdS:Mn luminescent nanocrystals," Advanced Functional Materials, vol. 14, no. 2, pp. 152-156, 2004.

[13] D. Kim, M. Miyamoto, and M. Nakayama, "Surfacemodification effects on luminescence properties of CdS and CdMnS quantum dots prepared by a reverse-micelle method," Physica Status Solidi (c), vol. 0, no. 4, pp. 1233-1236, 2003.

[14] N. Karar, H. Chander, and S. M. Shivaprasad, "Enhancement of luminescent properties of $\mathrm{ZnS}: \mathrm{Mn}$ nanophosphors by controlled ZnO capping," Applied Physics Letters, vol. 85, no. 21, pp. 5058-5060, 2004.

[15] D. Haranath, H. Chander, N. Bhalla, P. Sharma, and K. N. Sood, "Surface distribution studies and improved photoluminescence characteristics of silica coated $\mathrm{ZnS}: \mathrm{Mn}$ nanophosphor layers," Applied Physics Letters, vol. 86, no. 20, Article ID 201904, 3 pages, 2005.

[16] S. R. Cordero, P. J. Carson, R. A. Estabrook, G. F. Strouse, and S. K. Buratto, "Photo-activated luminescence of CdSe quantum dot monolayers," Journal of Physical Chemistry B, vol. 104, no. 51, pp. 12137-12142, 2000.

[17] P. Calandra, A. Longo, and V. T. Liveri, "Synthesis of ultrasmall $\mathrm{ZnS}$ nanoparticles by solid-solid reaction in the confined space of AOT reversed micelles," Journal of Physical Chemistry $B$, vol. 107, no. 1, pp. 25-30, 2003.

[18] S. Santra, H. Yang, P. H. Holloway, J. T. Stanley, and R. A. Mericle, "Synthesis of water-dispersible fluorescent, radioopaque, and paramagnetic CdS:Mn/ZnS quantum dots: a multifunctional probe for bioimaging," Journal of the American Chemical Society, vol. 127, no. 6, pp. 1656-1657, 2005.

[19] H. Bao, Y. Gong, Z. Li, and M. Gao, "Enhancement effect of illumination on the photoluminescence of water-soluble CdTe nanocrystals: toward highly fluorescent CdTe/CdS core-shell structure," Chemistry of Materials, vol. 16, no. 20, pp. 38533859, 2004.

[20] N. Gaponik, D. V. Talapin, A. L. Rogach, et al., “Thiol-capping of CDTe nanocrystals: an alternative to organometallic synthetic routes," Journal of Physical Chemistry B, vol. 106, no. 29, pp. 7177-7185, 2002.
[21] A. Shavel, N. Gaponik, and A. Eychmüller, "Efficient UVblue photoluminescing thiol-stabilized water-soluble alloyed ZnSe(S) nanocrystals," Journal of Physical Chemistry B, vol. 108, no. 19, pp. 5905-5908, 2004.

[22] M. Ando, C. L. Li, and N. Murase, "Photoluminescence properties and zeta potential of water-dispersible CdTe nanocrystals," Materials Research Society Symposium Proceedings, vol. 789, pp. 123-128, 2004.

[23] H. Yang, P. H. Holloway, G. Cunningham, and K. S. Schanze, "CdS:Mn nanocrystals passivated by ZnS: synthesis and luminescent properties," Journal of Chemical Physics, vol. 121, no. 20, pp. 10233-10240, 2004. 

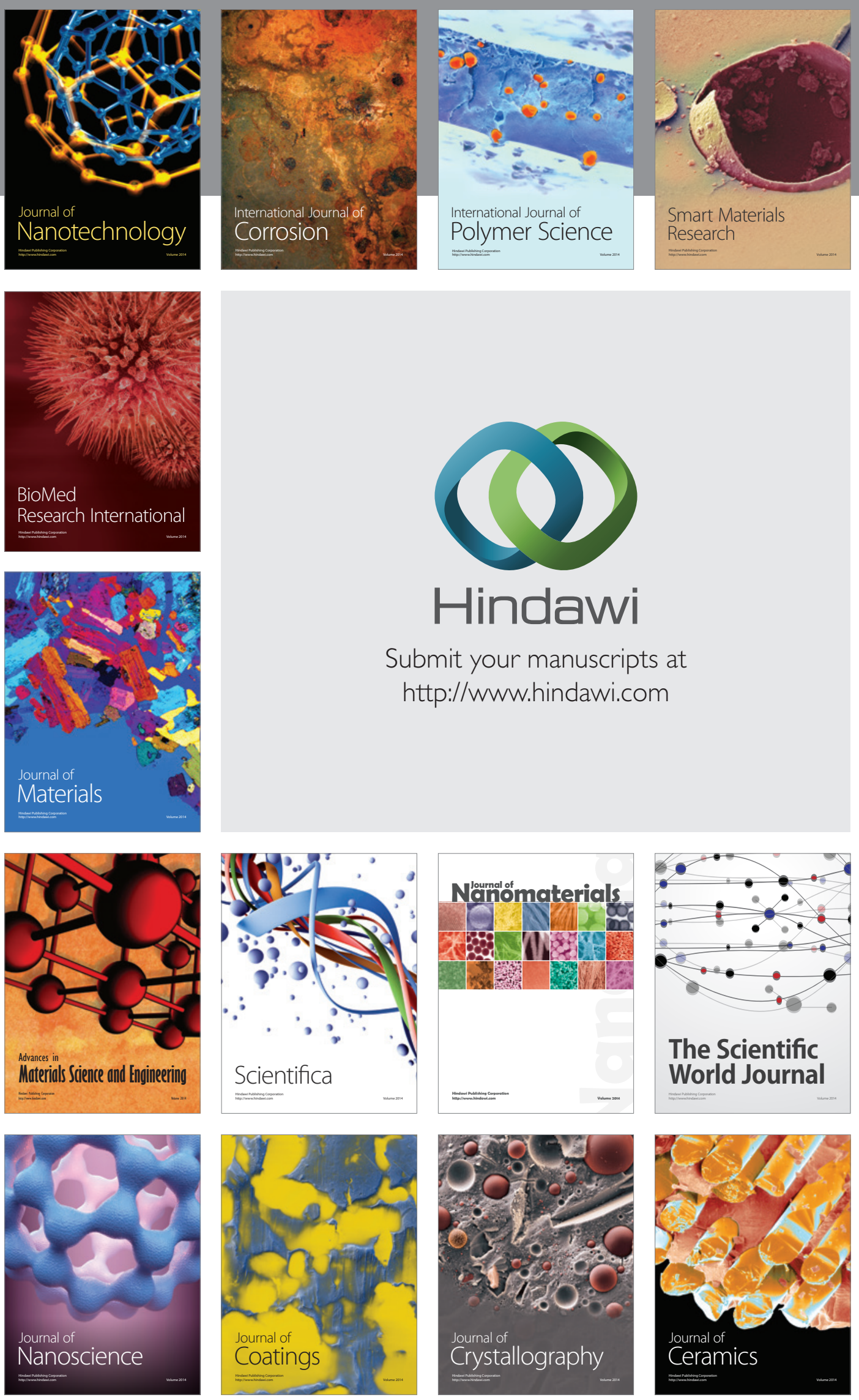

The Scientific World Journal

Submit your manuscripts at

http://www.hindawi.com

\section{World Journal}

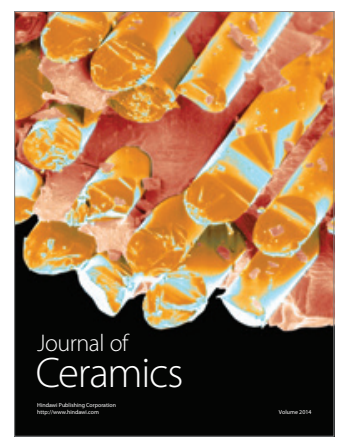

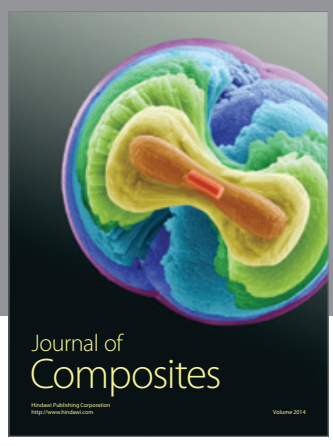
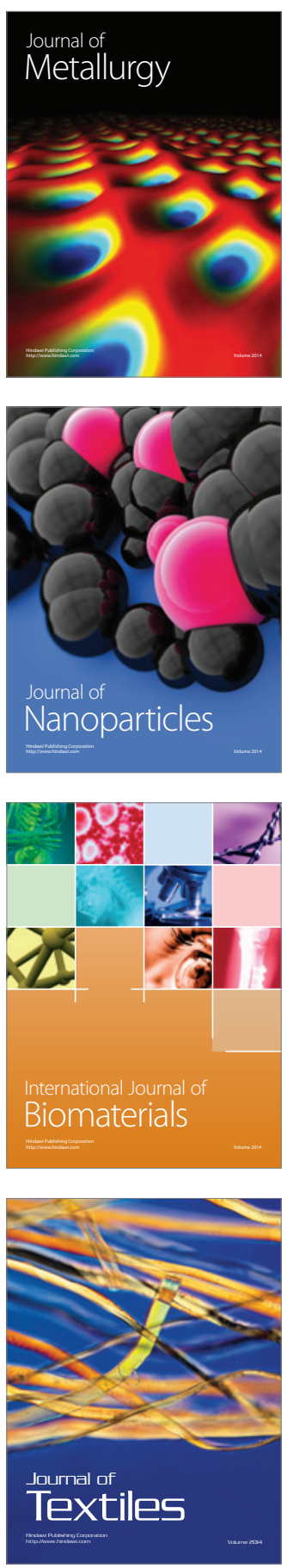